\title{
Aesthetic gingival management: Preservation of the anatomical structures and the gingival aesthetics by immediate implant-insertion after loss of anterior teeth and premolars-Results of a 5-year prospective study with 348 inserted one-phase implants
}

\author{
Angelo Troedhan ${ }^{1}$, Izabela Schlichting ${ }^{1}$, Andreas Kurrek ${ }^{2}$ \\ ${ }^{1}$ Center for Facial Esthetics Vienna, Vienna, Austria \\ ${ }^{2}$ Implantology Clinic Ratingen, Ratingen, Germany \\ Email: troed@aon.at
}

Received 23 March 2013; revised 22 April 2013; accepted 8 May 2013

Copyright (C 2013 Angelo Troedhan et al. This is an open access article distributed under the Creative Commons Attribution License, which permits unrestricted use, distribution, and reproduction in any medium, provided the original work is properly cited.

\begin{abstract}
Implant insertion is an alternative to classical treatment with bridges after tooth extraction or traumatic tooth loss in the anterior and premolar region of the upper and lower jaw. Nevertheless both types of prosthetic treatment inhere major gingival aesthetic pitfalls by physiological vertical and horizontal resorption. Aim of the study was to investigate if the immediate insertion of root analogue single-stage implants and prosthetic treatment could prevent gingival recessions and bone resorption thus keeping the natural gingival aesthetics untouched. Between 2003 and 2006, 348 root analogue Q1-Implants (TRINON Karlsruhe $\mathrm{GmbH} / \mathrm{Germany}$ ) were inserted in 342 patients's premolar and molar regions immediately after tooth extraction or traumatic tooth loss and treated with provisional resin crowns. The observation period with recurring 6-month clinical and radiographic check-ups was a minimum of 5 years. Of 348 inserted implants $4(1.15 \%)$ were lost resulting in an overall success rate of $98.85 \%$. In the first 12 weeks after surgery a mean recession of the buccal gingival margin of $0.2 \mathrm{~mm}$ (SD 0.34 ) could be detected, after final prosthetic treatment an overall mean recession of $0.2 \mathrm{~mm}$ (SD 0.13) within the survey period. Immediate implant insertion of root analogue single stage implants and immediate prosthetic treatment with provisional resin crowns after minimal invasive tooth extraction seems to be appropriate to prevent bone resorptions of the buccal alveolar crest and the recessions of the gingiva and papillae and
\end{abstract}

thus suggests to be the preferable treatment to keep the natural gingival and papillary aesthetics untouched preventing further traumatic surgical gingival reconstructions.

Keywords: Oral Implantology; Single Phase Implants; Immediate Implant Insertion; Gingival Aesthetics

\section{INTRODUCTION}

Due to the increasing acceptance and demand by patients for tooth implants as an alternative to bridges after tooth loss, a predictable treatment and long-term cosmetic aspects are the main focus in the area of anterior teeth and premolars.

Causes for potential loss of anterior and premolar teeth in majority are:

1) Immediate traumatic loss of one or more anterior teeth (accident);

2) After-effects of dental trauma due to necessity of root treatment (root resorptions, increasing discolouring, formation of granulomas caused by longitudinal root cracks, fatigue cracking, sub-gingival crown fractures);

3) Non-sustainable anterior or premolar teeth after root treatment (granuloma recurrence after apisectomy, fatigue cracking in longitudinal root direction, sub-gingival crown fracture);

4) Periodontal resorption caused by periodontitis (generic, hygienic deficits, imperfect dentin-crown margins of inserted crowns).

Patients having lost anterior and/or premolar teeth caused by 1 to 3 could still be treated with bridges but 
prosthetical treatment-indication shifts more and more towards implant-insertion, particularly in cases when adjacent teeth fail as potential bridge posts because they are either fully intact (no fillings) or already prosthetically treated with crowns or already serve as bridge posts for inserted bridges.

Tooth loss caused by 4) must be carefully considered whether the patient is verifiably willing to observe the hygienic requirements correctly or whether a prosthetic implant can meet the cosmetic requirements with regard to the gingival soft tissues especially in the anterior maxillary region ("pink aesthetics").

Both treatment alternatives-bridges and implantsare critical from the physiological point of view regarding the preservation of the individual hard and soft tissues of the alveolar ridge and by this the preservation of the individual natural gingival aesthetic at the tooth-loss site [1].

After traumatic tooth loss (without fracture-related loss of the buccal alveolar ridge lamella or the entire alveolar ridge) and surgical tooth removal (without iatrogenic bone defects) a centripetal reduction of the alveolar crest both in width (narrowing) and height will inevitably occur in the course of bone-healing of the alveola which can be compensated only partly by augmentational procedures known as "socket preservation" [2].

These physiological procedural resorptions of hard tissues followed by gingival recessions often lead to difficulties in the recovery of the patient-specific gingival and papillary aesthetics in the course of prosthetic treatment and might lead to the necessity of highly demanding and traumatic gingival aesthetic surgery with not always fully satisfactory results $[3,4]$ or lately the gingival and papillary application of hyaluronic acid to improve the cosmetic results [5] with limited applicability.

Cases being treated merely by bridge prosthetics carry the risk of unmanageable gap-development between the pontic/pontics and the gingiva and/or gingival papilla recessions with exposure of the crown margins towards the pontic site immediately when the prosthetic treatment has been carried out too early (mostly before the end of the 3rd month after tooth loss), in the long term by centripetal and vertical atrophy of the toothless alveolar ridge site below the pontic/pontics [6-11].

A delayed implant insertion or the use of two-stage implants might lead to similar resorptive effects with adverse effects regarding gingival aesthetics demanding secondary complex mucogingival surgery $[3,4]$. These techniques (mucogingival shift- or rotation-flaps, free connective tissue transplants etc.) require routine and a high grade of surgical skills and inhere another risk of medical and/or esthetical complications.

Aim of this prospective study was to verify whether immediate implant-insertion of single-stage implants after tooth loss (traumatic/extraction) in the anterior and pre- molar region could be suitable to prevent aesthetic deficits of the gingival margins and papillae short and long term and to quantify the success rate over 5 years.

\section{MATERIALS \& METHODS}

From 2003 to 2006 the authors have appointed all patients eligible for immediate implant insertion after tooth loss in the anterior and premolar region based on clinical and radiographical examinations by meeting the following general criteria:

1) Traumatic loss of one or more teeth without loss of parts or the entire corresponding alveolar crest;

2) Sequelae of dental trauma due to the necessity of root treatment (root resorptions, increasing discolouring, formation of granulomas caused by longitudinal root cracks, fatigue cracking, sub-gingival crown fractures);

3) Non-sustainable tooth after root treatment (granuloma recurrence after apisectomy, fatigue cracking in longitudinal root direction, sub-gingival crown fracture);

4) Approximal plaque index in the area of premolars and anterior teeth of max. $40 \%$ (corresponding to the average individual oral hygiene indices in the population).

Patients with acute or chronic periodontitis of single teeth with loosening degree 2 and 3 and periodontal alveolar ridge defects of more than $1 / 3$ of the root length or generalised periodontitis were excluded.

The single-stage and root-analogue Q1-implant system (Trinon Karlsruhe GmbH/Germany) was chosen as implant system for this study.

The single-stage Q1-implant (Figure 1) provides all required implant diameters and lengths (diameters: 3.5/4.5/5.6 mm, lengths: 8/10/12/14/16/18 mm, gingival spacer: $2 / 4 \mathrm{~mm}$ ) corresponding to the anatomy of anterior and premolar roots and it provides a self-cutting screw

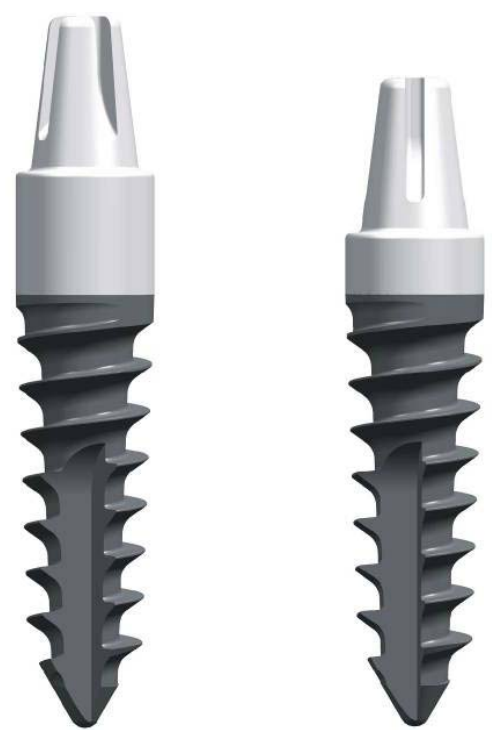

Figure 1. Q1 root-analogue implants. 
design with progressive bone-compacting effects for sufficient primary stability.

It allows intrasurgical flexibility regarding a variable insertion depth independent from prior implant bed preparation and correction of the insertion angle (Figure 2).

Once elected by the general criteria patients had to meet potential implant-site specific criteria:

a) the crestal mesio-distal diameter of the alveola had to be smaller than the diameter of the implant's gingival shaft of the available Q1-implants (3.5, 4.5 and $5.6 \mathrm{~mm}$ );

b) apical of the apex of the tooth-loss site or extractionalveola another minimum of $3 \mathrm{~mm}$ of alveolar bone had to be depicted in the radiograph;

c) a radiographic verified granuloma needed to be smaller than the implant's diameter;

d) the gingiva, gingival margin and papillae needed to be intact and free of inflammation (Figures 3 and $\mathbf{4}$ ).

The surgical protocol included the following mandatory steps:

1) Clean incision of the cervical junction epithelium down to the alveolar crest with a scalpel;

2) Atraumatic tooth extraction preferably with extraction pliers or periotomes (after 2005 with ultrasonic surgical ligament cutters) (Figures 5 and $\mathbf{6}$ );

3) Transalveolar curettage of existing granulomas with sharp excavators (after 2005 with ultrasonic surgical currettes);

4) Measurement of extracted root length and final determination of the implant length and implant diameter;

5) Pilot drill along root axis (in the upper anterior al-

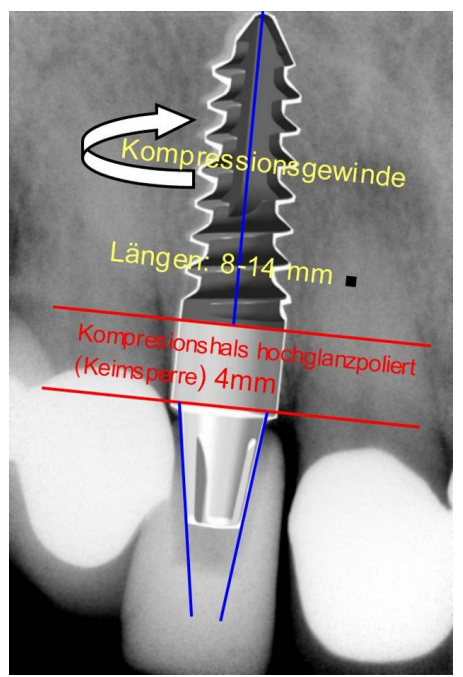

Figure 2. Q1-implants allow variable insertion-depths and angles at final insertion and can be inserted up to $4 \mathrm{~mm}$ deeper and corrected by angle up to $7^{\circ}$ than prior drilling preparation and thus be precisely adapted to the gingival margin and the alveola at final insertion.

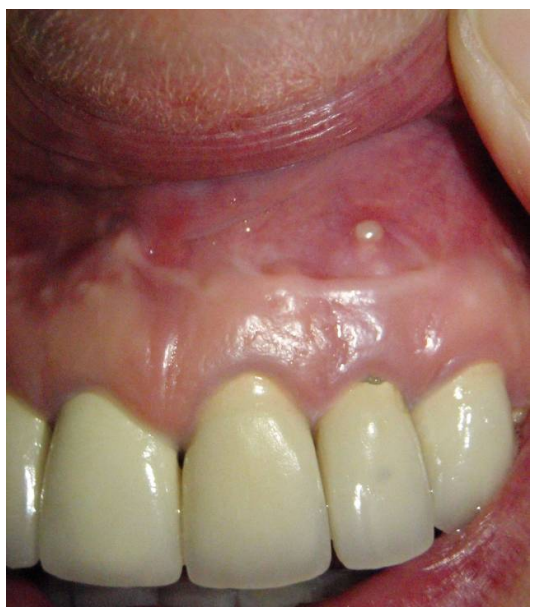

Figure 3. Case 1: Recurrence of an apical granuloma of the second left incisor with a chronic fistula after two apisectomies. Adjacent gingiva and papillae are free of inflammation, adjacent teeth are treated with intact metalloceramic crowns.

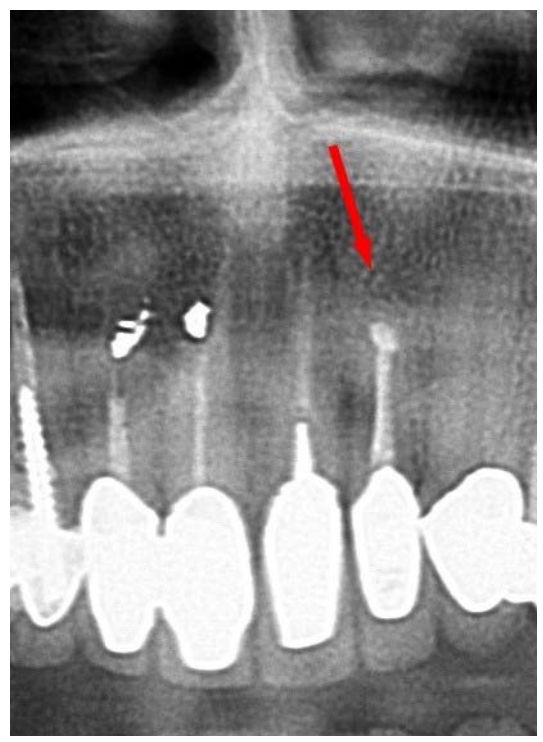

Figure 4. Case 1: Corresponding radiograph. Apical granuloma recurrence marked with a red arrow.

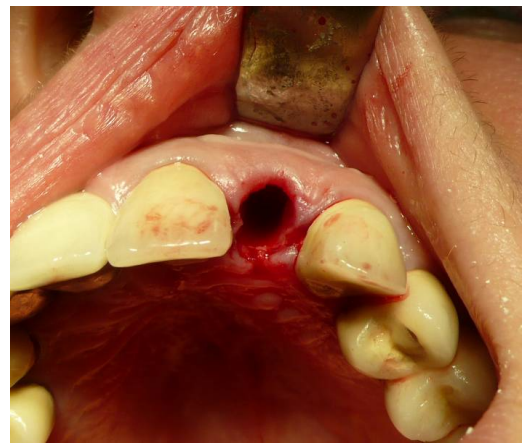

Figure 5. Case 1: Extraction site. 


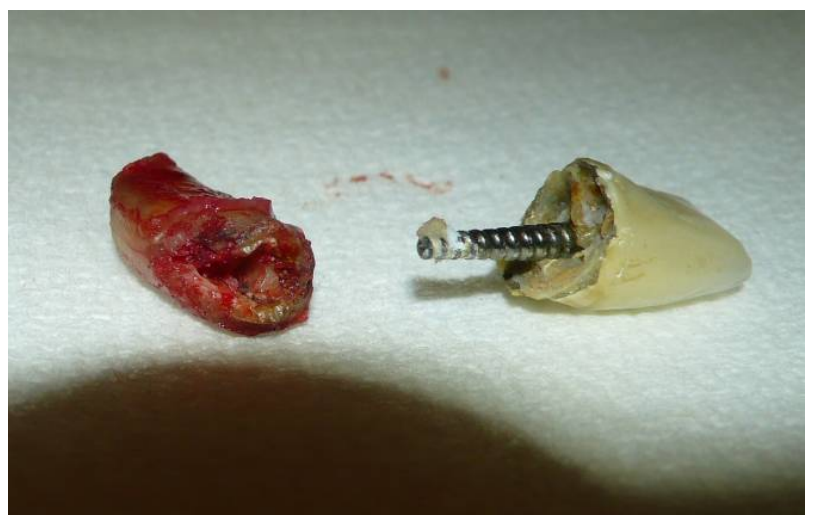

Figure 6. Case 1: Removed root ready for measurement of axial length.

veolar crest: orientation to the palatal alveolar bone, in the upper premolar region in the palatal root socket (if any), in the lower alveolar crest in the central axis of the root (Figure 7);

6) Preparation of the final implant bed in the upper alveolar crest with bone-condensers/spreaders (Q-tome), in the lower alveolar crest with form drills up to the final implant diameter;

7) Insertion of the implant/implants at least 1.5 - $2 \mathrm{~mm}$ below the buccal gingival margin or up to $1 \mathrm{~mm}$ subcrestal related to the buccal alveolar bone (measured from the prosthetic platform for maintaining the biologic width) (Figures 8 and 9);

8) Fabrication and insertion of an individually shaped and for the emergence profile anatomically correctly designed resin provisional crown based on the prefabricated snap-on Q-caps (Figure 10) after surgery as "wound closure". Fixation of the provisional with temporary cement and adaptive sutures was prohibited in order to prevent additional traumatization of the gingival seam and not to change the natural gingival emergence profile iatrogenically (Figure 11).

All patients were shielded after surgery for a period of 4 to 5 days with Clindamycin $300 \mathrm{mg} 3 \times 1$ /day or Amoxicillin/Clavulic Acid 1 g $2 \times 1$ /day and interviewed 7 days after surgery about pain and swelling. The final prosthetic treatment with ceramometallic or $\mathrm{ZiO}$-crowns was carried out 3 to 4 months after surgery (Figure 12). Patients were advised and trained by a dental hygienist to apply thoroughly tooth brushing by the "red-white"-technique with soft tooth-brushes and to strictly avoid horizontal tooth brushing.

Follow-up examinations were conducted over a period of a minimum of 5 years and parameters were collected and documented every six months in check-ups as follows:

1) Measurement of the distance between the incisal edge of the temporary resin crown and the buccal gingival margin in the midline of the crown with a micrometer

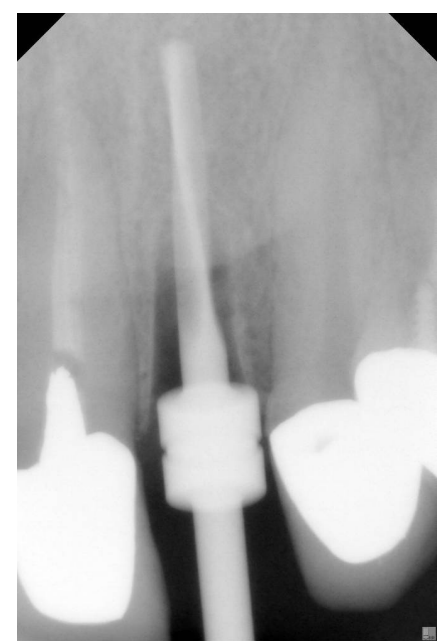

Figure 7. Case 1: Intrasurgical $\mathrm{X}$-ray with pilot-drill in site.

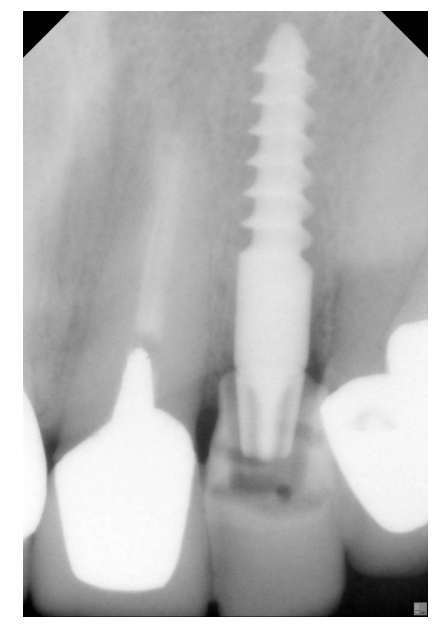

Figure 8. Case 1: Post-surgical X-ray with Q1-implant (Diameter $3.5 \mathrm{~mm}$, screw-length: 12 $\mathrm{mm}$, gingival neck: $4 \mathrm{~mm}$ ) and anatomical correct full functional provisional resin crown in site.

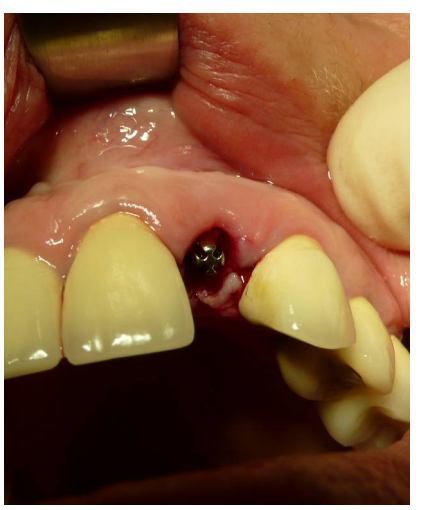

Figure 9. Case 1: Implant site immediately after surgery. 


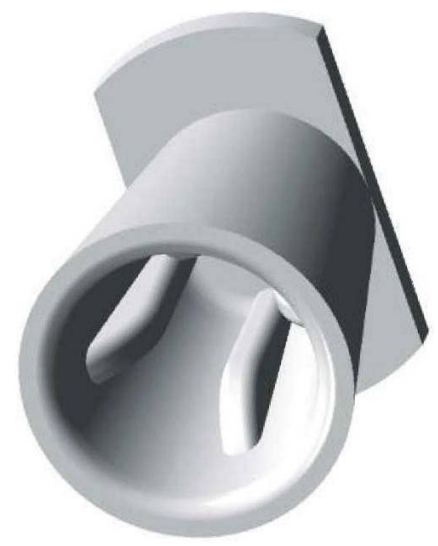

Figure 10. Q-Cap as base for fabrication of a full functional provisional resin crown.

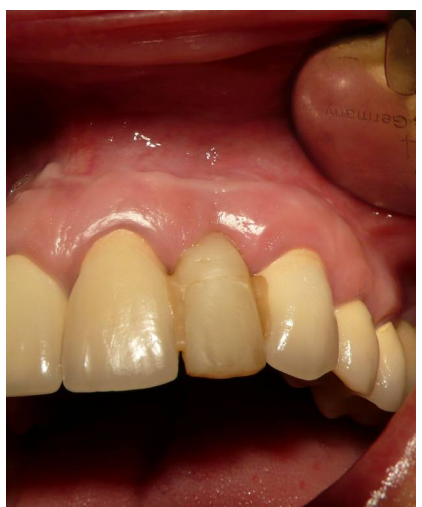

Figure 11. Case 1: Fully functional anatomically correct provisional resin crown clipped onto implant and stabilized with flow-composite to the adjacent crowns. Provisional is not cemented to prevent additional traumatization of the gingival margin and papillae at cement removal.

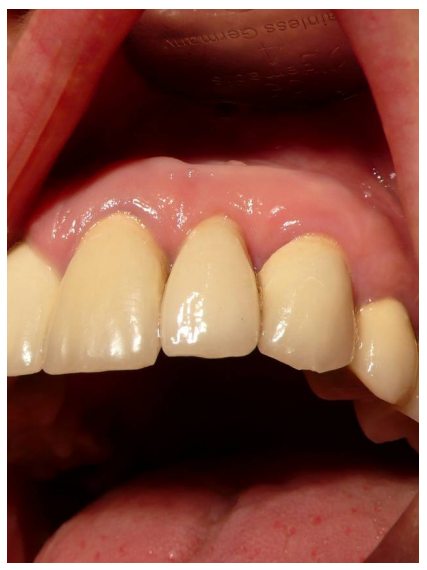

Figure 12. Case 1: Final ceramometallic crown in site. every 4 weeks after surgery;

2) Measurement of the distance between the incisal edge of the final ceramometallic or ZiO-crown and the buccal gingival margin in the midline of the crown with a micrometer every 6 months;

3) Clinical assessment of the peri-implant gingiva as compared to the natural dentition;

4) Radiographic check and measurement regarding changes of the crestal periimplant-bone for resorptions;

5) Measurement of pocket depths, BOP;

6) Survey of the individual patients' satisfaction grade (patient's statements during the control examinations in the categories: very satisfied, satisfied, need to be improved, poor).

Gingival recessions were assessed statistically by mean value and Standard Deviation. Crestal resorptions were not assessed statistically due to lack of reproductible positioning of the digital X-Ray sensor and/or unreliable periodontal probing in split-millimeter scale.

\section{RESULTS}

A total of 362 out of 403 inspected patients (89.83\%) complied with the general eligibility-protocol as defined.

Of the 362 patients eligible 19 patients decided against implant treatment (5.25\%), one patient $(0.28 \%)$ had to be excluded due to an apical cyst with a larger diameter than the implant body.

Reasons for rejecting implant treatment were:

a) "Financial reasons": 11 patients (57.89\%);

b) "Bad experience with implants" reported by relatives or friends: 6 patients (31.58\%);

c) "Fear of allergy against implants/biological incompatibility": 2 patients (10.53\%).

In total 348 Q1-implants were inserted in 342 patients. 4 patients $(1.17 \%)$ were treated with implants and provisional prosthetics as emergency cases within 8 hours after anterior tooth loss due to trauma.

From a total of 348 inserted implants 4 implants in 3 patients were lost within the observation-period (loss rate: 1.15\%): 2 patients suffered from loss of one implant each (Sites: second and first incisior) within the 3-months healing period prior final prosthetic treatment caused by periimplantitis and consecutive loosening in the 8th and 10th week after surgery, one patient lost 2 implants in the maxillary premolar site in the fourth year after surgery caused by peri-implantitis due to a lack in the personal oral hygiene.

93.86\% of the patients stated to have been free of any pain and swelling after surgery, $6.14 \%$ reported mild pain and subjective swelling until the 4th day after surgery (Table 1).

All implants in site revealed consistent alteration-free buccal gingival conditions with classical stippling and in- 
Table 1. Statistical analysis of overall implant loss, implant loss pre- and postprothetic and pain and swelling occurrance.

\begin{tabular}{|c|c|c|c|c|}
\hline Pat. \# eligible & Pat. \# treated & Pat. \# with Implant loss & \# Implant loss preprosthetic & Postsurgical Pain/Swelling \\
\hline 362 & $342(100 \%)$ & $3(0.88 \%)$ & $2(0.57 \%)$ & None \\
\hline $\begin{array}{l}\text { Pat. \# Rejected implant } \\
\text { treatment }\end{array}$ & \# of implants inserted & \# of implants lost & \# Implant loss postprothetic & $321(93.86 \%)$ \\
\hline 19 & 348 (100\%) & $4(1.15 \%)$ & $2(0.57 \%)$ & Yes \\
\hline Pat. \# excluded & & & & $21(6.14 \%)$ \\
\hline 1 & & & & \\
\hline
\end{tabular}

tact papillary structure within the observation period and beyond.

Recessions of the buccal gingival margin of $1 \mathrm{~mm}$ up to a maximum of $1.8 \mathrm{~mm}$ occurred in $5.78 \%$ of the cases during the first 8 to 12 weeks after surgery. After insertion of the final crown(s) 3 - 4 months post surgery recessions of a maximum of $0.4 \mathrm{~mm}$ could be detected in the observation period of 5 years (Table 2).

In the radiographic follow-up the periimplant alveolar ridge structure was clinically consistently stable with minor vertical bone losses of a maximum of $1 \mathrm{~mm}$ in the observation period although measurement values in radiographs and periodontal probing for technical reasons were not reliable enough for statistical evaluation.

Pocket depth probing resulted consistently in values of 1.5 to $2 \mathrm{~mm}$ which is the natural biological width and a papillary bleeding index (PBI) of 0 to maximum 1 (Figure 13).

In the 5-year observation period there was no case of exposure of the implant-neck caused by gingival or bone recessions.

The long-term satisfaction was rated high by the patients:

$71 \%$ rated the cosmetic result as "very satisfactory" after 5 years post surgery,

$24 \%$ as "satisfactory",

$4 \%$ as "need to be improved", and only

$1 \%$ as "unsatisfactory".

The $4 \%$ patient group rating the long term cosmetic result as "needs to be improved", mainly criticized a light "bluish cast" of the buccal gingiva when exposed to bright artificial light.

\section{DISCUSSION}

The results of this prospective study suggest immediate implant-insertion of a suitable root-analogue single stage implant after extraction or traumatic tooth loss to significantly provide excellent functional and aesthetic longterm results. This might be attributed both to the atraumaticity of the surgical protocol preventing damage of vital tissue structures, regenerative physiological entities and biological functional units [12-19] and the use of the chosen Q1-implant system which proved it's easy and
Table 2. Statistical analysis of gingival recession behaviour before and after final prosthetic treatment.

\begin{tabular}{cccc}
\hline $\begin{array}{c}\text { Gingival recessions 8th - } \\
\text { 12th week post surgery }\end{array}$ & $\mathrm{mm}$ & $\begin{array}{c}\text { Gingival recessions after } \\
\text { final prosth. treatment }\end{array}$ & $\mathrm{mm}$ \\
\hline mean & 0.2 & mean & 0.2 \\
$\min$ & 0.1 & $\min$ & 0.0 \\
$\max$ & 1.8 & $\max$ & 0.4 \\
$\mathrm{SD}$ & 0.34 & $\mathrm{SD}$ & 0.13 \\
\hline & & & \\
& & & \\
& & & \\
& & &
\end{tabular}

Figure 13. Case 1: Clinical situation after 5.3 yr.

predictable application and which seems to be suitable particularly for this indication due to it's root analogue compression screw design granting a high primary stability at insertion and physiological masticatory force distribution into the alveolar bone (Figures 13-22).

The duration of treatment of 3 - 4 months until the insertion of the final crown is similar to a treatment with bridges provided the healing period after tooth extraction is respected to avoid subpontical gaps and aesthetic compromises such as papillary atrophy ("black triangles") of the adjacent teeth.

The significant clinical success rate regarding the function and gingival aesthetics is also reflected by the patients' appreciation of not having to wear any removable dentures during the interim period or to bear the high costs of interim bonding bridges as well as the overall simplicity of the treatment with a full natural aesthetic and functional outcome which some patients described as "if it would be my own natural tooth”. 


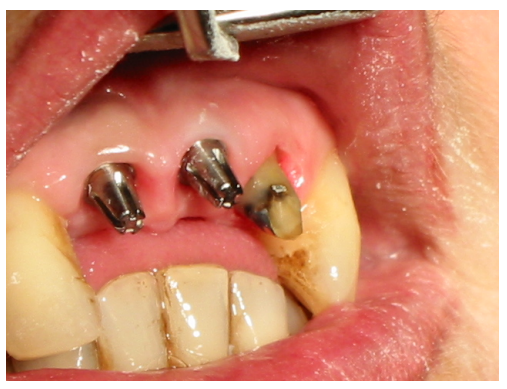

Figure 14. Case 2: Implant insertion after traumatic tooth loss (2003).

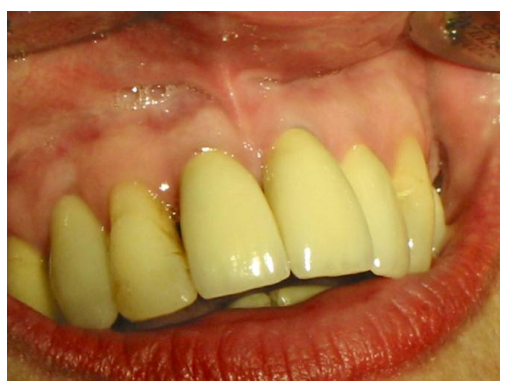

Figure 15. Case 2: Clinical situation after 1 yr.

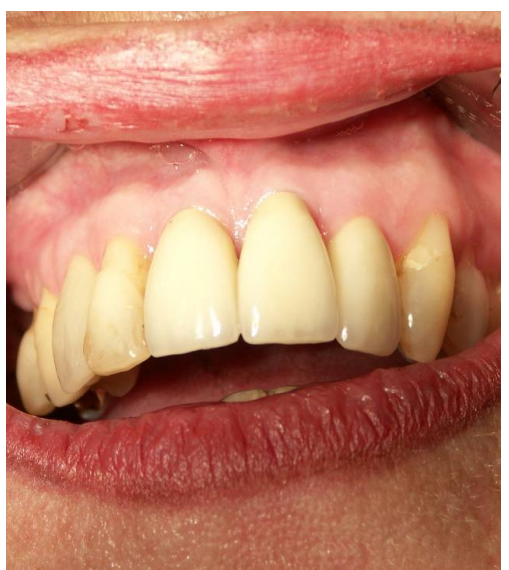

Figure 16. Case 2: Clinical situation after 3 yr.

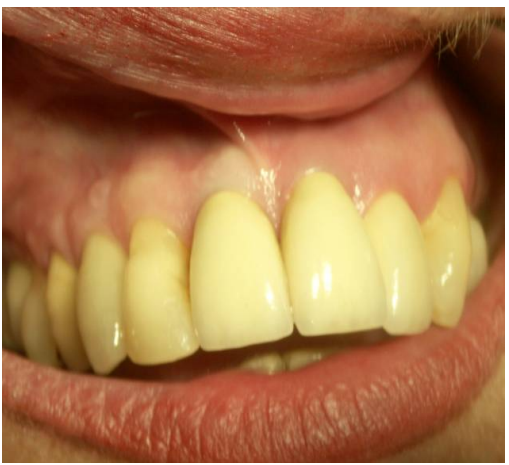

Figure 17. Case 2: Clinical situation after $5.8 \mathrm{yr}$.

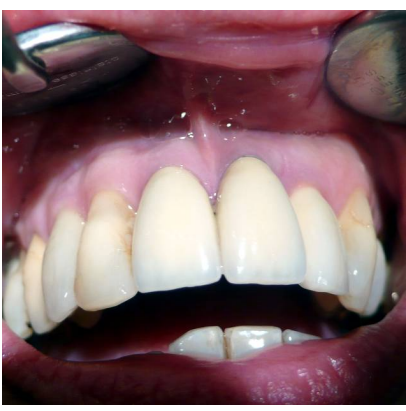

Figure 18. Case 2: Clinical situation after $9.6 \mathrm{yr}$ (recession of the gingiva at the left first incisor starts to reveal the implant neck but generally follows the recession pattern of the natural dentition).

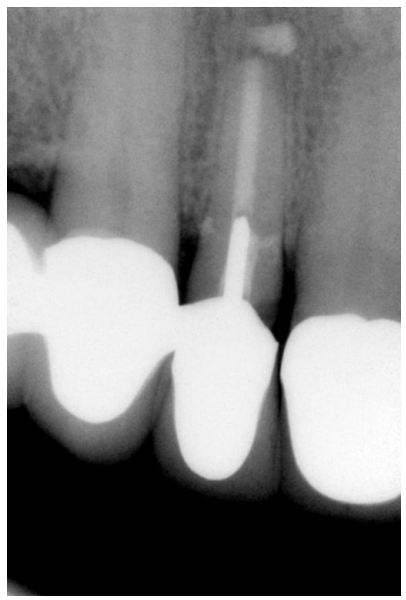

Figure 19. Case 3: Presurgical situation: horizontal root fracture of right 2nd incisor, neighbouring teeth act as posts for bridges.

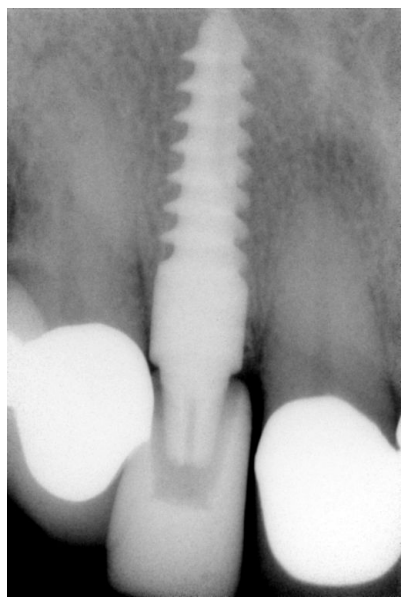

Figure 20. Case 3: Post surgical X-ray with implant and provisional resin crown in site. 


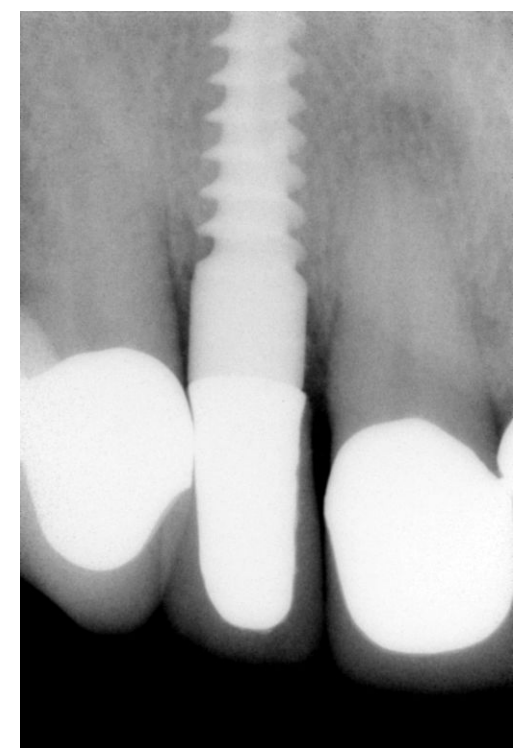

Figure 21. Case 3: Final prosthetic treatment after 3.5 months.

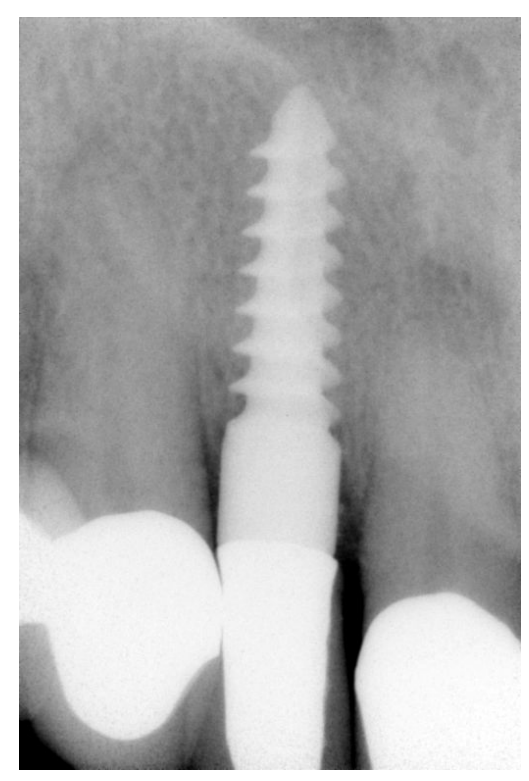

Figure 22. Case 3: X-ray 5.6 yr after treatment: No significant crestal bone resorptions can be detected.

It could not be verified whether the slight postsurgical pain which was stated by $6 \%$ of the patients resulted from the application of the local anaesthetic, the extractionprocedure or implant placement. However even for this patient group the endured pain was within the subjectively expected range.

Since the minimal invasive procedure of tooth extraction and simultaneous insertion of a single-stage root-analogue implant can be applied in $90 \%$ of the cases conservative therapy with bridges can be considered as "therapy of second choice” in the future. No cosmetic compromises and/or complex and complication-bearing secondary soft tissue reconstructions need to be carried out when the described implant therapy is applied.

In summary the authors therefore conclude that the immediate implant-insertion after tooth extraction with a root-analogue single stage implant in the area of anterior teeth and premolars with immediate loading by an anatomically correct provisional crown is the therapy of choice for the preservation of the bony alveolar ridge structures and gingival aesthetics when complied with the indication criteria. The treatment process is efficient, cost effective and burdens patients as little as possible.

\section{REFERENCES}

[1] Cawood, J.I. and Howell, R.A. (1991) Reconstructive preprosthetic surgery: I. Anatomical considerations. International Journal of Oral and Maxillofacial Surgery, 20, 75-82. doi:10.1016/S0901-5027(05)80711-8

[2] Bartee, B.K. (2001) Extraction site reconstruction for alveolar ridge preservation. Part 1: Rationale and materials selection. Journal of Oral Implantology, 27, 187-193. doi:10.1563/1548-1336(2001)027<0187:ESRFAR >2.3.C $\underline{\mathrm{O} ; 2}$

[3] Roccuzzo, M., Bunino, M., Needleman, I. and Sanz, M. (2002) Periodontal plastic surgery for treatment of localized gingival recessions: A systematic review. Journal of Clinical Periodontology, 3, 178-194.

[4] Oates, T.W., Robinson, M. and Gunsolley, J.C. (2003) Surgical therapies for the treatment of gingival recession. A systematic review. Annals of Periodontology, 8, 303320. doi:10.1902/annals.2003.8.1.303

[5] Becker, W., Gabitov, I., Stepanov, M., Kois, J., Smidt, A. and Becker, B.E. (2010) Minimally invasive treatment for papillae deficiencies in the esthetic zone: A pilot study. Clinical Implant Dentistry and Related Research, 12, 1-8. doi:10.1111/j.1708-8208.2009.00247.x

[6] Stein, R.S. (1966) Pontic-residual ridge relationship: A research report. Journal of Prosthetic Dentistry, 16, 251285. doi:10.1016/0022-3913(66)90080-1

[7] Schropp, L., Wenzel, A., Kostopoulos, L. and Karring, T. (2003) Bone healing and soft tissue contour changes following single-tooth extraction: A clinical and radiographic 12-month prospective study. The International Journal of Periodontics and Restorative Dentistry, 23, 313- 323.

[8] Denissen, H.W., Kalk, W., Veldhuis, H.A. and van Waas, M.A. (1993) Anatomic considerations for preventive implantation. The International Journal of Oral \& Maxillofacial Implants, 8, 191-196.

[9] Schropp, L., Kostopoulos, L. and Wenzel, A. (2003) Bone healing following immediate versus delayed placement of titanium implants into extraction sockets-A prospective clinical study. The International Journal of Oral \& Maxillofacial Implants, 18, 189-199.

[10] Kalk, W., Denissen, H.W. and Käyser, A.F. (1993) Preventive goals in oral implantology. International Journal 
of Dentistry, 43, 483-491.

[11] Wheeler, S.L., Vogel, R.E. and Casellini, R. (2000) Tissue preservation and maintenance of optimum esthetics: A clinical report. The International Journal of Oral \& Maxillofacial Implants, 15, 265-271.

[12] Fugazzotto, P.A. (2002) Simplified technique for immediate implant insertion into extraction sockets: Report of technique and preliminary results. Implant Dentistry, 11, 79-82. doi:10.1097/00008505-200201000-00018

[13] Quirynen, M., Van Assche, N., Botticelli, D. and Berglundh, T. (2007) How does the timing of implant placement to extraction affect outcome? The International Journal of Oral \& Maxillofacial Implants, 22, 203-223.

[14] Kan, J.Y.K. and Rungcharassaeng, K. (2003) Interimplant papilla preservation in the esthetic zone: A report of six consecutive cases. The International Journal of Periodontics and Restorative Dentistry, 23, 249-259.

[15] Kois, J.C. (2001) Predictable single tooth periimplant es- thetics: Five diagnostic keys. Compendium of Continuing Education in Dentistry, 22, 199-208.

[16] Kan, J.Y.K., Rungcharassaeng, K. and Lozada, L. (2003) Immediate placement and provisionalization of maxillary anterior single implants: 1-year prospective study. The International Journal of Oral \& Maxillofacial Implants, 18, 31-39.

[17] Kan, J.Y.K., Rungcharassaeng, K., Umezu, T. and Kois, J.C. (2003) Dimensions of peri-implant mucosa: An evaluation of maxillary anterior single implants in humans. Journal of Periodontology, 74, 563-568. doi:10.1902/jop.2003.74.4.557

[18] Spear, F.M. (1999) Maintenance of the interdental papilla following anterior tooth-removal. Practical Periodontics and Aesthetic Dentistry, 11, 21-28.

[19] Garber, D.A., Salama, M.A. and Salama, H. (2001) Immediate total tooth replacement. Compendium of Continuing Education in Dentistry, 22, 210-218. 